\title{
Vascular changes in the lungs on the plain radiograph of the chest
}

\author{
I. H. KERR \\ M.B., M.R.C.P., F.F.R. \\ Consultant Radiologist, Brompton Hospital, London, S. W.3
}

\begin{abstract}
Summary
The normal anatomy of the pulmonary vessels is considered and correlated with the angiographic appearances.
\end{abstract}

Some pathological conditions causing an alteration in the vascular pattern as seen on the plain films are considered and the changes are described.

It is concluded that an appreciation of these changes in vascular pattern may indicate to the clinician the presence of local or distant disease.

\section{Introduction}

Up to as recently as 1942 there was difference of opinion as to the nature of the shadows in the lung fields in normal radiographs, some authors still considering them to be due to the bronchi. However, Lodge in 1946 stated emphatically 'that the linear shadows seen transversing the lung fields in normal radiographs are produced by the blood-filled arteries and veins of the lung'. Further he concluded that many of the lobar and segmental arteries, and main pulmonary veins could be identified in the normal plain chest radiograph.

Since that time the study of the pulmonary vasculature both in the normal and the abnormal has been greatly enhanced by pulmonary arteriography and there is a greater appreciation of the vascular changes in disease seen on the plain radiograph. Interpretation of the chest radiograph is now considerably dependent on the identification of vascular changes, especially in the absence of abnormal pulmonary shadows.

\section{Normal anatomy of pulmonary vessels Arteries}

So that proficiency in the assessment of the vascular pattern can be achieved, the observer should be familiar with the normal anatomy of both the pulmonary arterial tree, and the pulmonary veins. There are several normal variations described (Boyden, 1955; Jefferson, 1965). The intrapulmonary arteries are closely applied to the bronchi, though there are a greater number of variations in the origins of the lobar and segmental vessels than in those of the equivalent bronchi. Variations of arterial branching are greatest in the left upper lobe where there is no true typical pattern of branching. Beyond the origin of the segmental arteries, however, vessels remain intimately related to the bronchial axial pathways.

On the right side, the right pulmonary artery reaches the lung by passing in front of the right descending bronchus, between the upper and middle lobe bronchi. It gives off its largest branch (the truncus anterior) to the upper lobe, and then descends on the lateral aspect of the bronchus as the pars interlobaris until the middle lobe and apical segment of lower lobe arteries take origin. From this point the artery continues to descend as the pars basalis, on the lateral side of the bronchus, to the basal segments of the lower lobe (see Fig. 1).

The left pulmonary artery arches backwards over the left main bronchus, as the pars superior. It then descends, as on the right side, as the pars interlobaris, and then pars basalis, but on this side lying posterolateral to the bronchus. There is no definite truncus anterior on the left side, the upper lobe being supplied by a variable number of smaller branches arising from the outer aspect of the left pulmonary artery as it curves posteriorly and downwards over the left bronchus.

The arteries mainly give to the two hilar shadows on the plain frontal radiograph their characteristic shapes though that on the right is partly composed of the descending upper lobe veins (Lavender \& Doppman, 1962). The right hilar shape is that of a wide angled ' $V$ ', with the apex pointing to the left. One arm of the ' $V$ ' is derived from the pars interlobaris and the pars basalis, and the other the truncus anterior and descending upper lobe vein. On the left side the hilum is shaped like a long fat comma, being produced by the shadows of the pars superior, pars interlobaris and pars basalis of the left pulmonary artery with the small upper lobe arteries arising from the outer curve of the comma.

There is gradual diminution of the calibre of the artery shadows in proportion to the distance from the hila. The vessels are normally straight or gently curved, but not tortuous. The lower lobe vessels are fatter than the upper lobe vessels, as there is more 

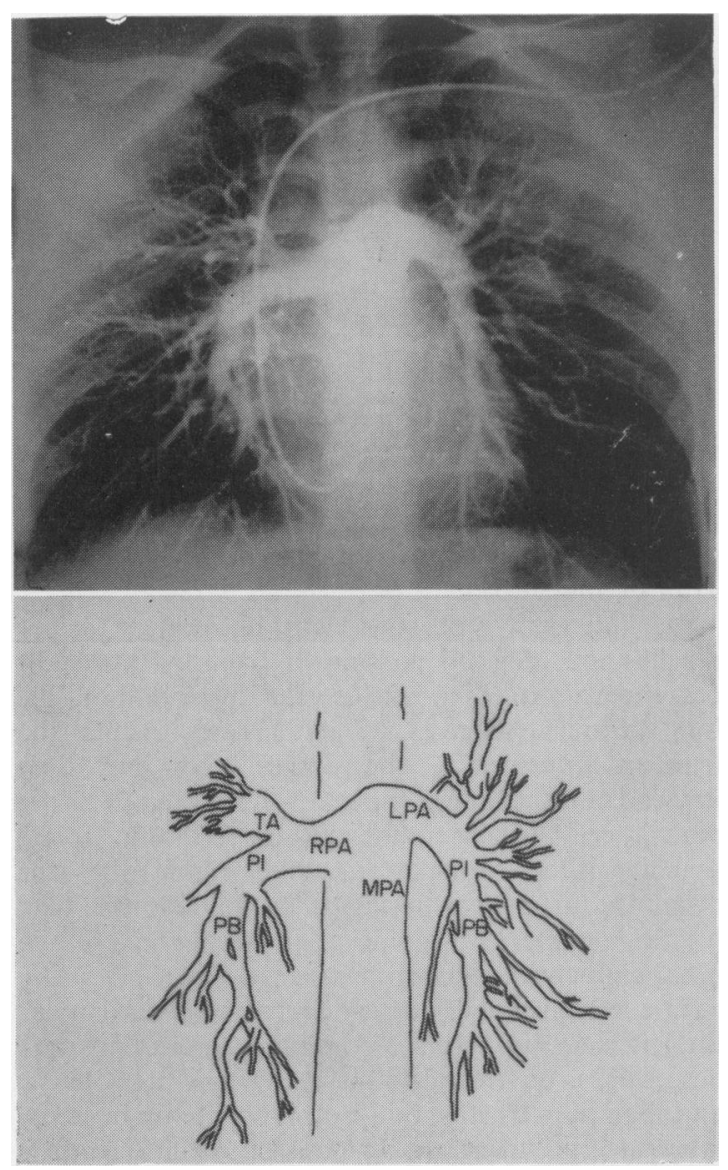

FIG. 1. Normal pulmonary arteriogram. MPA, main pulmonary artery; RPA, right pulmonary artery; LPA, left pulmonary artery; TA, truncus anterior; PI, pars interlobaris; PB, pars basalis.

lung parenchyma to supply. Any branch which arises has a diameter less than the stem, but the sum of the branch diameters exceeds that of the stem diameter. Towards the end of the arterial pathway regular branches at about $1 \mathrm{~cm}$ and then $1 \mathrm{~mm}$ may be observed, comparable to the centimetre and millimetre pattern described in the peripheral bronchogram (Reid \& Simon, 1958). Injection of postmortem specimens, however, reveals a great disparity between the branching patterns of the pulmonary artery and bronchial tree due to the presence of numerous supernumerary arterial branches, not visible on an angiogram in life (Elliott \& Reid, 1965).

\section{Veins}

Variation in the pattern of the veins is also seen, though like the arteries they follow closely the segmental portions of the bronchial tree. The variations are mainly in the larger vessels near the hila. In the lungs the vessel-bronchi relationship can be stated as a law, namely that artery, bronchus and vein are found in that order proceeding anticlockwise in the right lung and in the same order but clockwise in the left.

On the right side, the segmental veins from the middle and lower lobes join to form the inferior pulmonary vein which passes horizontally and medially into the left atrium, and is often easily identified on the plain radiograph below the hilar shadow of the right pulmonary artery. The veins of the right upper lobe vary in number but descend in a medial direction, crossing the pars interlobaris of the right pulmonary artery at the hilum, and joining to enter the upper right border of the left atrium just below the right hilum (Fig. 2).
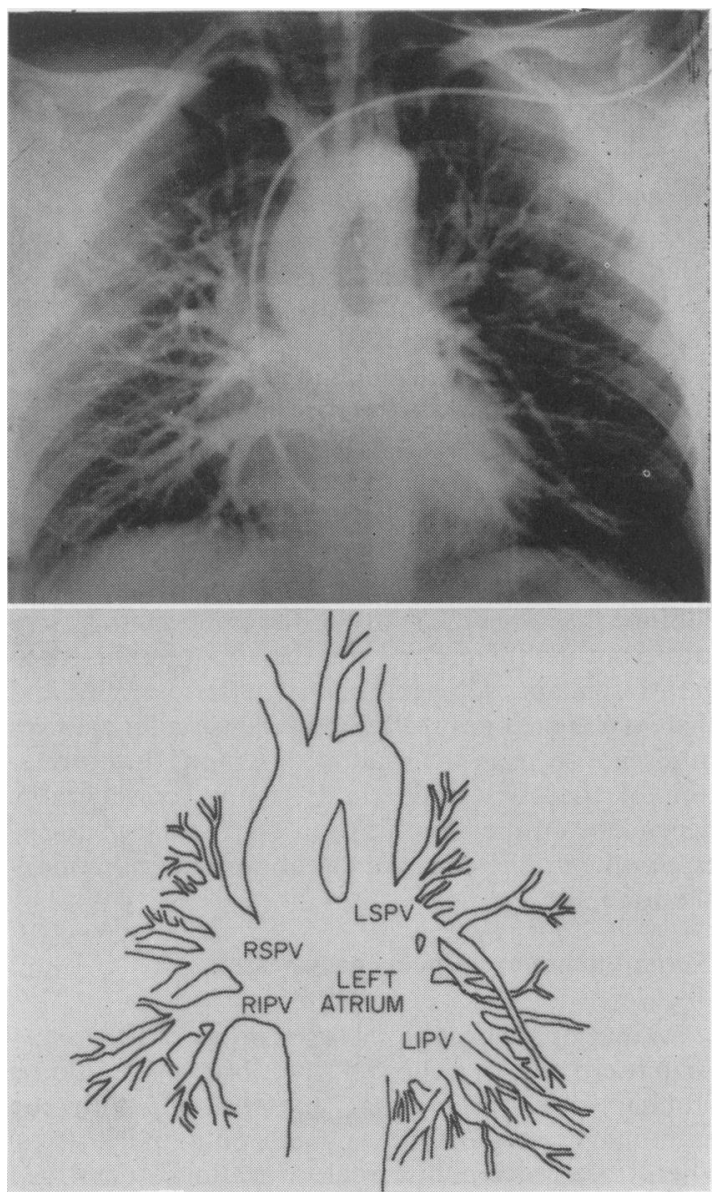

FIG. 2. Normal pulmonary venogram. Contrast medium is also seen in the left side of the heart and aorta. RSPV, right superior pulmonary vein; LSPV, left superior pulmonary vein; RIPV, right inferior pulmonary vein; LIPV, left inferior pulmonary vein. 
In the left lung the venous drainage is very similar to that on the right with the segmental vessels joining to form a superior pulmonary vein and an inferior pulmonary vein, which enter the left atrium.

It is the direction of the larger veins which makes them identifiable on the plain radiograph. They pass medially to the left atrium 1 or 2 in. below the hilar shadows produced by the large trunks of the left and right pulmonary arteries, and therefore, cross the branches of the arteries as these leave the hila.

As with the arteries, in the erect position, the veins of the upper lobes are smaller than those of the lower lobes. In the supine position the upper lobe veins fill with blood so that there is less difference in size between them and the lower lobe veins, though they still are smaller.

\section{Changes in the normal vascular pattern}

There are few diseases of the lungs which do not cause an alteration in some way, to the normal vessel pattern on the plain chest radiograph. The vessels are visible because blood and vascular tissue are denser than the air in the surrounding alveoli. If there is no air in the alveoli, either because of pulmonary collapse or consolidation, the vessels become invisible and cannot be identified.

Thus any condition which causes loss of air in the alveoli, will cause a loss of the normal vascular pattern. Such diseases embrace a large portion of pulmonary pathology and it is not the intention of this paper to provide an exhaustive list of pulmonary diseases which cause alteration of the normal vascular pattern, but rather to give some examples of the way in which observation of vascular change aids in the interpretation of the plain chest film.

\section{(1) Displacement of vessels}

Displacement of vessels in the lung indicates that some portion of the lung is reduced in volume. Space occupying lesions such as distension cysts or bullae compress the adjacent lung with displacement of vessels.

Observing the displacement of the hila is of help in identifying collapse of the lung. Thus in collapse of the left lower lobe (Fig. 3) the left hilum loses its normal round outer contour, as the pars interlobaris and pars basalis are displaced medially and downwards behind the upper part of the shadow of the heart. The left hilar shadow is normally slightly higher than the right, and in left lower lobe collapse it is displaced inferiorly to lie about the same level as that on the right or lower. The vessels in the upper lobe become splayed out as the upper lobe undergoes compensatory emphysema and the upper lobe becomes more transradiant. Similar changes are seen in right lower lobe collapse.

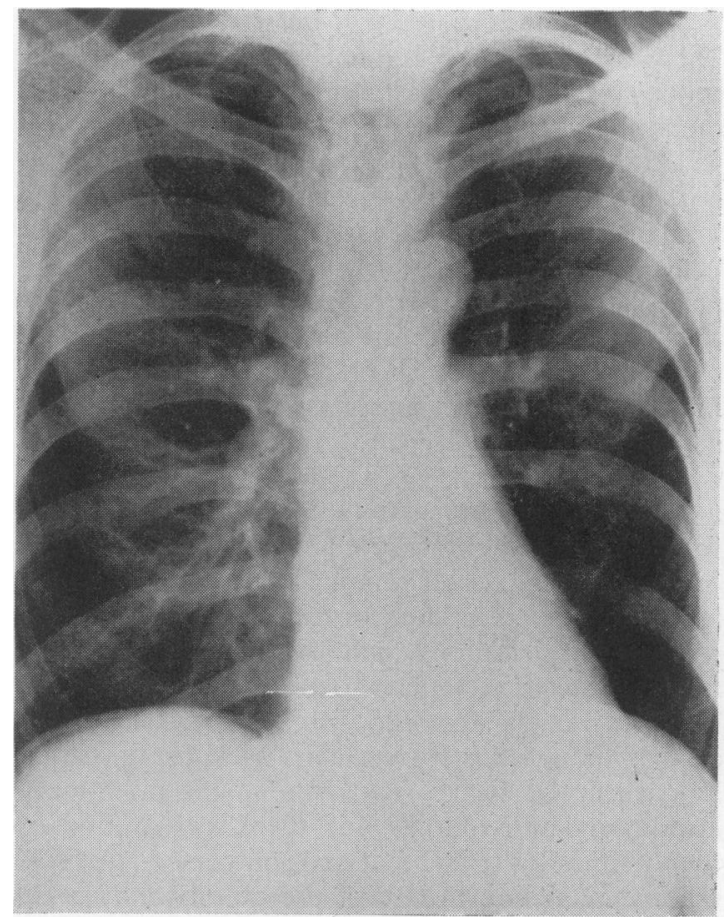

Fig. 3. Post-operative collapse of left lower lobe. The left pulmonary artery is displaced downwards so that the left hilum is small, and the normal round contour of the hilum is lost.

In upper lobe collapse or contraction the reverse occurs, and there is elevation of the hilar shadows. In long-standing disease such as tuberculosis it is the displacement of the vessel shadows coupled with displacement of the mediastinal structures that indicates fibrosis has taken place. An angular elevation of the hilar shadow with streaks of fibrosis leading to the apex of the lung is the typical appearance in long-standing upper lobe pulmonary tuberculosis.

\section{(2) Decrease in vascularity of lung}

Decrease in vacularity of the lung, either local or general, may be very difficult to appreciate. Knowledge of the normal appearances of the vessels is essential. Only after careful study of the vascular pattern in normal radiographs can the abnormal be detected. Radiographic technique is critical. An over-exposed film may suggest oligaemia, and an under-exposed film plethora of the lung fields. Rotation of the patient may make one lung field appear oligaemic in comparison with the other. If the radiograph has not been taken on full inspiration the vessels appear more conspicuous, especially in the lower lung fields. 
(a) Generalized decrease of pulmonary vascularity. There is generalized pulmonary oligaemia in cyanotic congenital heart disease, usually the result of pulmonary stenosis or atresia, or tricuspid atresia in which a right to left shunt is present. The normal bronchial artery supply to the lung is usually so small as to be invisible on the plain radiograph, but under these circumstances, the bronchial arteries may dilate to such an extent as to be visible (Fig. 4). They then produce a mottled pattern in the perihilar regions. This may be so marked as to obscure the fact that the pulmonary vessels are smaller than normal.

Acquired generalized oligaemia is seen in pulmonary emphysema (Fig. 5). The radiological features of emphysema have been described by Laws \& Heard (1962) and Simon (1964). The mid-lung pulmonary arteries appear narrow, straight and sometimes of uniform calibre over several centimetres instead of tapering towards the periphery. The finer vessels are reduced so that there is a rather clear homogeneous transradiant background to the lung field. In several cases the main pulmonary artery becomes enlarged and there is dilatation of the hilar vessels. In the normal person there is gradual reduction in size of the pulmonary vessels from the hilum to the periphery, but in emphysema there is a rather abrupt reduction in size between the hilar vessels and the mid-lung vessels. Other features of emphysema, the low flat diaphragm, large retro-

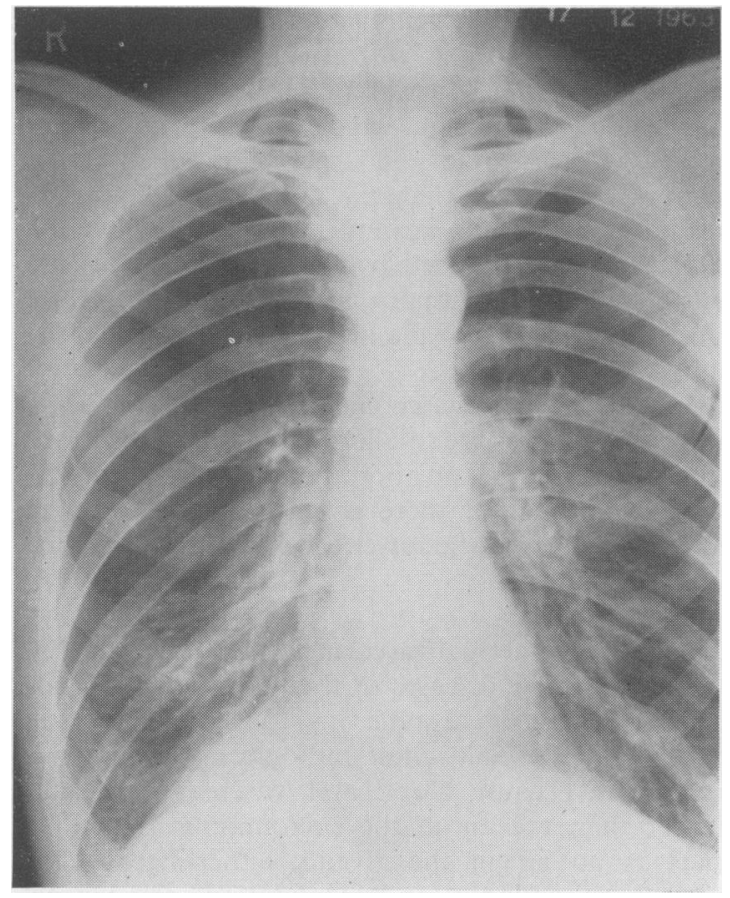

FIG. 5. Emphysema. There is marked reduction in the pulmonary vessel size in the mid lung field, particularly in the right upper and mid zones. sternal translucent zone, the narrow vertical heart,

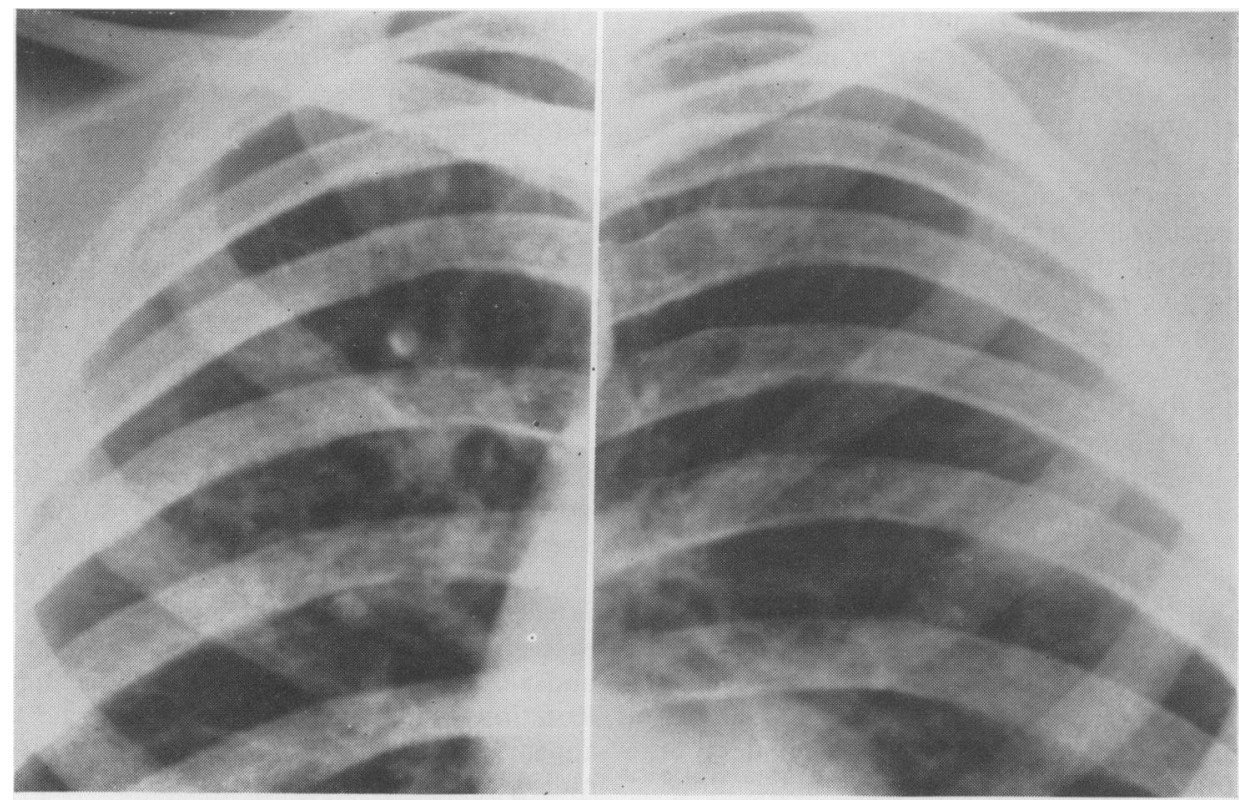

FIG. 4. Pulmonary atresia. No pulmonary arteries are seen. The vessel shadows are irregular, and are produced by bronchial arteries and a few small pulmonary veins. 
and bullae, may be present. Patients with asthma uncomplicated by emphysema may have radiological signs of air trapping, low flat diaphragms, transradiant lungs, and narrow vertical heart, but the intrapulmonary vessels, unlike those in emphysema, are normal in size and not narrow. In primary pulmonary hypertension the main pulmonary artery and hilar vessels are large, and there is reduction in size of the intrapulmonary vessels, but unlike emphysema there is no evidence of air trapping; the diaphragm is normal.

(b) Localized oligaemia. Congenital absence of one pulmonary artery is rare but provides a characteristic radiographic appearance. The affected lung is reduced in volume but appears normally aerated. There is an absence of the normal vascular shadow at the hilum and in place of the branching system of blood vessels normally seen, there is the network of smaller vessels, giving an irregular pattern, typical of enlarged bronchial arteries. These are the only vessels supplying blood to the lung (Good, 1961).

Similar appearances are seen in hypoplasia of a pulmonary artery (Belcher \& Pattinson, 1957) though in most of these cases there are associated abnormalities of the bronchial tree.

In unilateral transradiant lung (Macleod's syndrome), there is a uniform reduction in pulmonary vasculature throughout the affected lung, or portion of lung. It is now considered that this condition is probably due to arrested growth of the lung due to severe infection in childhood (Reid et al., 1967).

The observation in pulmonary embolism of oligaemia of the lung goes back to Westermark in 1938, and was much disputed for several years. In both acute and chronic pulmonary embolism the affected area, whether segment, lobe or entire lung may show oligaemia (Fleischner, 1962). Localized oligaemia is easier to detect than widespread oligaemia since a reduction in size of the vessels in one or more zones can be detected by reference to the vessel size in other zones. The changes are most easily recognized in chronic pulmonary embolic disease, though the radiograph may appear misleadingly normal in these patients (Chrispin, Goodwin \& Steiner, 1963). However, careful study of plain radiographs taken in acute pulmonary embolism, particularly if massive, reveals detectable oligaemia in nearly all cases (Figs. 6 and 7). Shadows due to infarcts are present in only about two-thirds of patients with pulmonary embolism (Kerr, Simon $\&$ Sutton, 1969). The quality of the radiograph is obviously of the utmost importance in assessing these changes in patients who are frequently seriously ill, and often unable to sit up. However, with good technique and using modern high-powered portable units a satisfactory film should be obtainable.

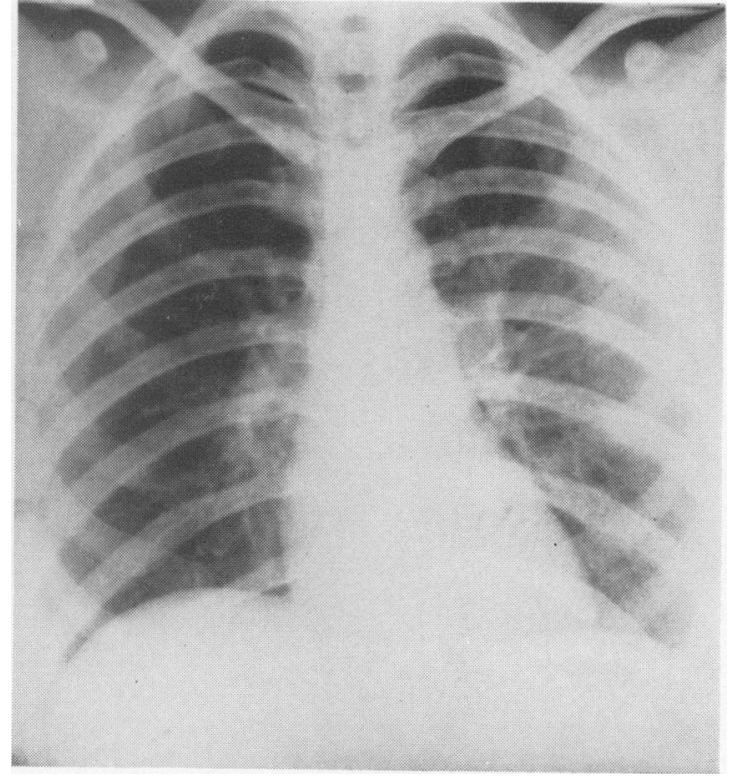

FIG. 6. Acute massive pulmonary embolism. Plain chest radiograph showing reduced vascularity of right lung. Note particularly loss of small vessel pattern in the periphery of the lung as compared with the left.

\section{(3) Increase in pulmonary vascularity}

(a) Generalized. Astley (1959) reviewed some of the radiological signs provided by the plain radiograph and fluoroscopy, in congenital heart disease. He divided the patients into acyanotic and cyanotic groups. The presence of pulmonary plethora in the acyanotic patients indicates a left to right shunt, either via a septal defect, or a patent ductus, or anomalous pulmonary veins. Pulmonary plethora in cyanotic patients suggests the possibility of transposition, truncus arteriosus, infantile co-arctation or the Eisenmenger syndrome.

In patients with Eisenmenger syndrome where there is reversal of left to right shunt, the largest hearts and the biggest proximal pulmonary arteries are seen in association with atrial septal defects (Rees \& Jefferson, 1967). The plain radiograph is often almost normal in ventricular septal defect with reversed shunt particularly in older children.

Pulmonary plethora may be acquired as the result of a septal defect, either following infarction of the septum or traumatic rupture. The onset of plethora is sudden and it may be difficult to distinguish from pulmonary congestion due to left heart failure in these usually very ill patients, but if there has been the sudden development of a loud systolic murmur the possibility of a septal defect should be considered.

In some cases of chronic bronchitis, Group 3 (syn. 'blue bloater', cor pulmonale), described by Scarrow 


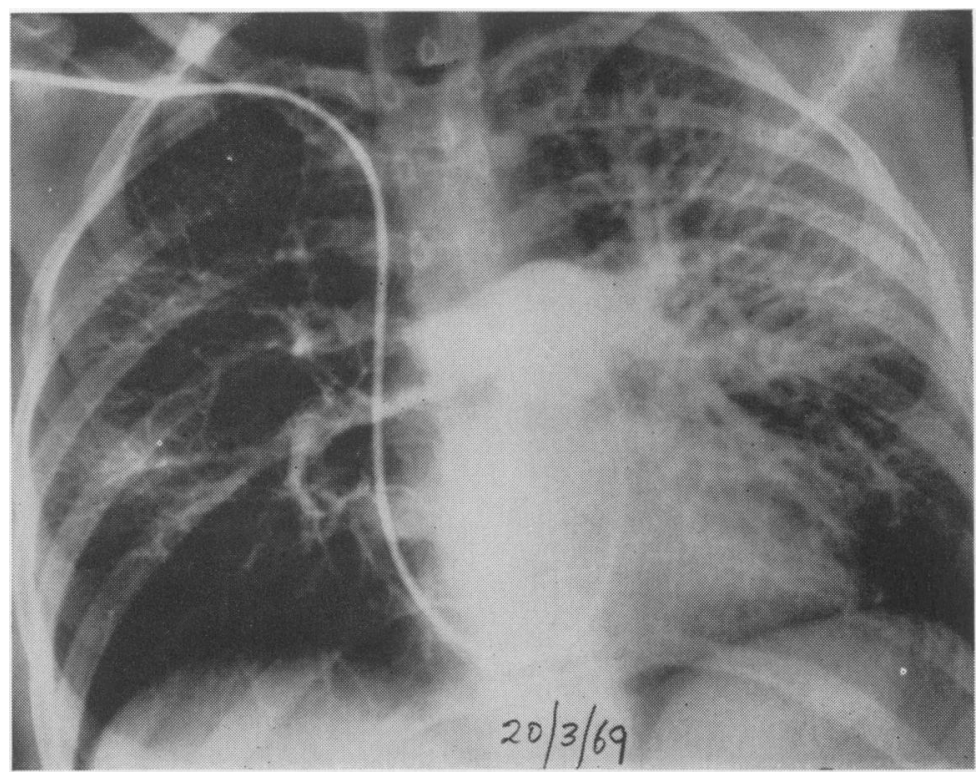

Fig. 7. Acute massive pulmonary embolism. Pulmonary arteriogram of the same patient as Fig. 6. showing embolism in the right main pulmonary artery.

(1966) where there is no evidence of emphysema, there may be enlargement of the intrapulmonary arteries. Unlike the other groups, in which there is emphysema, a more normal type of terminal arborization of the pulmonary arteries is seen.

In polycythaemia, not secondary to heart or respiratory disease, there is frequently an increase in the size of the small pulmonary vessels in the periphery of the lung. There may be a mottling in the mid and lower zones of the lungs present, due to a large number of small peripheral vessels seen end-on (Pitman, Steiner \& Szur, 1961).

Patients with cirrhosis of the liver may show small ill-defined nodular shadows in the lungs, predominantly in both lower zones. Berthelot et al. (1966) inspected post-mortem lung specimens in thirteen patients with cirrhosis, six of whom had nodular shadows. They showed small angiomata on the pleural surfaces, looking like the spider naevi seen on the skin in such patients. There was filling of an excessive number of peripheral small branches of the pulmonary arteries within the respiratory portion of the lung. The authors suggest that the nodular shadows might well arise from widespread dilatation of these small vessels.

(b) Local. Where there is diminution of vascularity of the lungs of a patchy nature, localized hyperaemia may occur in the normal areas of lung. This can be seen in pulmonary emphysema, particularly of the bullous variety, and also in pulmonary embolism.
There are two types of pulmonary arterio-venous fistula, both of which cause abnormally large vessels (Good, 1961). The first type, the more common, is between a pulmonary artery and a pulmonary vein. The second type is rare, and is between the systemic circulation and the pulmonary vessels. The first, if severe enough, may produce cyanosis, as it causes a right to left shunt. The second is acyanotic as the blood flows from the oxygenated systemic circulation to the pulmonary circulation. There is usually a round or oval shadow in the periphery of the lung connected to the hilum by two large vessels. One of these is an artery and the other a vein, and usually their connections can be identified, if not on the plain radiograph, by tomography (Fig. 8). Multiple fistulae are present in $30-40 \%$ of cases. A bruit may be heard over the lesion. Some of the patients with pulmonary arterio-venous fistulae suffer from hereditary haemorrhagic telangiectasia in whom ruby coloured lesions may be seen on the skin and mucous membranes.

Pulmonary arterio-venous fistula should be distinguished from partial anomalous venous drainage, in which there is an anomalous course of the pulmonary veins. In both conditions a large vein may be visible, but in arterio-venous fistula two vessels should be visible, the draining vein and the feeding artery. In anomalous venous drainage one large vein is seen and it receives tributaries, giving it a branched appearance. Anomalous veins of the right lung may drain into the left atrium, the right atrium, the hepatic vein or either vena cava. Veins 


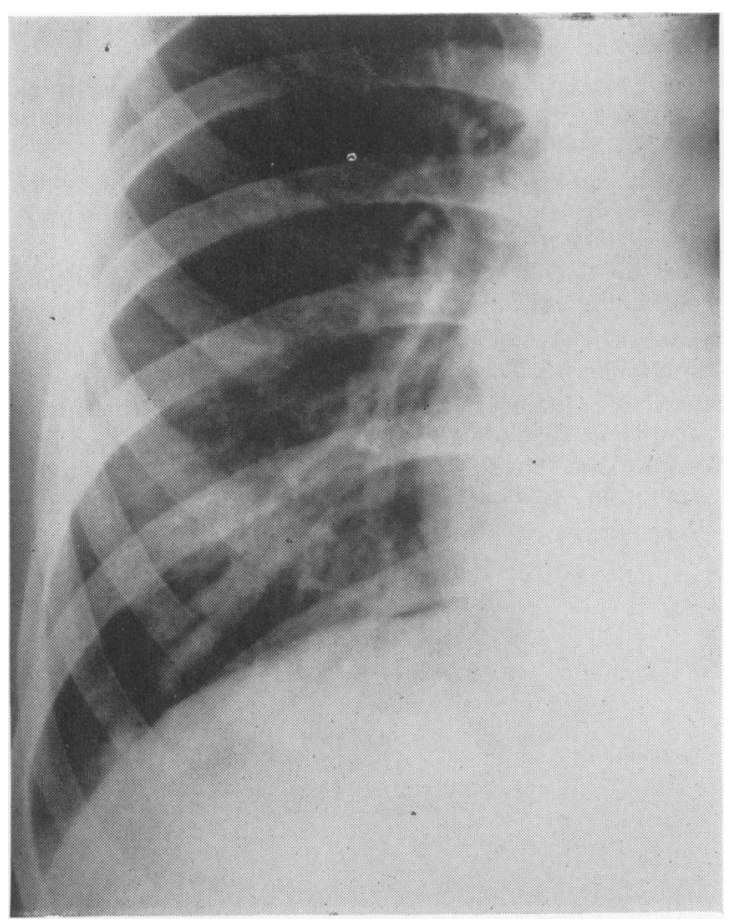

Fig. 8. Arterio-venous fistula in the right lower lobe. Note the two vessels, the artery and the vein leading to and from the lesion in the costo-phrenic sulcus.

in the left lung may drain into the left innominate vein. The commonest of these is seen as a large vessel running vertically in the right lung, gathering tributaries, and passing through the diaphragm near the right cardiophrenic angle.

\section{(4) Changes in pulmonary vasculature resulting from pulmonary venous hypertension}

Simon (1958) demonstrated that in patients with mitral stenosis there is engorgement of the upper lobe veins, and narrowing of lower lobe veins, and that the changes in vascularity provide some indication of the severity of pulmonary venous hypertension. The upper lobe veins were graded by Simon. In the normal range (Grade 0 ) the veins are not conspicuous and are smaller than the arteries. In Grade 1 there is slight engorgement of the upper lobe veins which become easily identifiable by contrast to the arteries. They are one to one and a half times the normal width. In Grade 2 the main tributaries are obviously dilated two to three times normal. The small tributaries are also prominent and traceable to the periphery. In Grade 3 the venous trunks and major tributaries are markedly dilated, three to five times normal width.

Unfortunately in correlating physiological studies with the degree of dilatation of the upper lobe veins, it is found that there is not a direct proportional relationship. The appearances are affected by the pulmonary artery pressure. As pulmonary hypertension progresses there is enlargement of the main pulmonary artery and its proximal branches with diminution of the peripheral vessels, which become narrower and more tortuous. These changes affect the lower lobes first, then become widespread (Goodwin et al., 1955).

The combined effects of pulmonary venous hypertension and pulmonary arterial hypertension, are therefore to reduce the peripheral pulmonary blood flow so that the venous engorgement becomes less, thus explaining why some patients with severe mitral stenosis show only slight venous engorgement. Analysis of these changes on the plain radiograph, and correlation with physiological studies, shows that assessment attains a clinically valuable degree of accuracy (Milne, 1963).

\section{Conclusion}

Like observation of the hands, or of the mouth, in the physical examination of a patient, so the study of the vascular changes on the plain chest radiograph may provide the clinician with considerable information of both local and distant disease. The careful study of the vessels in the lungs may be rewarding in supplying both anatomical and physiological information.

\section{References}

Astley, R. (1959) Simple methods in the diagnosis of congenital heart disease. British Journal of Radiology, 32, 355.

Belcher, J.R. \& Pattinson, J.N. (1967) Hypoplasia of lobar pulmonary arteries: report of three cases. Journal of Thoracic Surgery, 34, 357.

Berthelot, P., Walker, J.G., Sherlock, S. \& Reid, L. (1966) Arterial changes in the lungs in cirrhosis of the liver-lung spider naevi. New England Journal of Medicine, 274, 291.

Boyden, E.A. (1955) Segmental Anatomy of the Lung. McGraw-Hill, New York.

Chrispin, A.R., Goodwin, J.F. \& Steiner, R.E. (1963) The radiology of obliterative pulmonary hypertension and thrombo-embolism. British Journal of Radiology, 36, 705.

Elliott, F.M. \& Reid, L. (1965) Some new facts about the pulmonary artery and its branching pattern. Clinical Radiology, 16, 193.

FleisChNeR, F.G. (1962) Pulmonary embolism. Clinical Radiology, 13, 169.

Good, C.A. (1961) Certain vascular abnormalities of the lungs. American Journal of Roentgenology, Radium Therapy, and Nuclear Medicine, 85, 1009.

Goodwin, J.F., Hunter, J.D., Cleland, W.P., Davies, L.G. \& SteINER, R.E. (1955) Mitral valve disease and mitral valvotomy. British Medical Journal, 2, 573.

JEFFERSON, K.E. (1965) The pulmonary vessels in the normal pulmonary angiogram. Proceedings of the Royal Society of Medicine, 58, 677.

Kerr, I.H., Simon, G. \& Sutton, G.C. (1969) Pulmonary embolic disease: the value of the plain radiograph. (In preparation.) 
LAVENDER, P. \& DopPMAN, J. (1962) The hilum in pulmonary venous hypertension. British Journal of Radiology, 35, 303.

LAWs, J.W. \& HEARD, B.E. (1962) Emphysema and the chest film: a retrospective radiological and pathological study. British Journal of Radiology, 35, 750.

LODGE, T. (1946) The anatomy of the blood vessels of the human lung as applied to chest radiology. British Journal of Radiology, 19, 1.

MACLEOD, W.M. (1954) Abnormal transradiency of one lung. Thorax, 9, 147.

MiLne, E.N.C. (1963) Physiological interpretation of the plain radiograph in mitral stenosis, including a review of criteria for the radiological estimation of pulmonary, arterial and venous pressures. British Journal of Radiology, 36, 902.

Pitman, R.G., Steiner, R.E. \& Szur, L. (1961) The radiological appearances of the chest in polycythaemia vera. Clinical Radiology, 12, 276.
ReEs, R.S.O. \& JefFerson, K.E. (1967) The Eisenmenger syndrome. Clinical Radiology, 18, 366.

Reid, L. \& Simon, G. (1958) The peripheral pattern in the normal bronchogram and its relation to peripheral pulmonary anatomy. Thorax, 13, 103.

Reid, L., Simon, G., Zorab, P.A. \& Seidelin, R. (1967) The development of unilateral hypertransradiancy of the lung. British Journal of the Diseases of the Chest, 61, 190.

SCARROW, G.D. (1966) The pulmonary angiogram in chronic bronchitis and emphysema. Clinical Radiology, 17, 54.

Simon, G. (1964) Radiology and emphysema. Clinical Radiology, 15, 293.

SIMON, M. (1958) The pulmonary veins in mitral stenosis. Journal of the Faculty of Radiologists, 9, 25.

WESTERMARK, N. (1938) On roentgen diagnosis of lung embolism. Acta radiologica, 19, 357. 